\title{
Bragg Grating Optical Filters by UV Nanoimprinting
}

\author{
M. Casalboni, ${ }^{1}$ L. Dominici, ${ }^{2}$ V. Foglietti, ${ }^{3}$ F. Michelotti, ${ }^{2}$ E. Orsini, ${ }^{1}$ \\ C. Palazzesi, ${ }^{1}$ F. Stella, ${ }^{1}$ and P. Prosposito ${ }^{1}$ \\ ${ }^{1}$ Department of Physics, University of Rome-Tor Vergata and Istituto Nazionale Scienze e Tecnologie dei Material (INSTM), \\ Via della Ricerca Scientifica, 1-00133 Rome, Italy \\ ${ }^{2}$ Department of Energetics, University of Rome - la Sapienza, and CNISM, Via A. Scarpa 16, 00161 Rome, Italy \\ ${ }^{3}$ CNR-IFN, Institute for Photonics and Nanotechnologies, Via Cineto Romano, 00156 Rome, Italy \\ Correspondence should be addressed to P. Prosposito, paolo.prosposito@roma2.infn.it
}

Received 22 November 2011; Accepted 28 December 2011

Academic Editor: Sevan P. Davtyan

Copyright () 2012 M. Casalboni et al. This is an open access article distributed under the Creative Commons Attribution License, which permits unrestricted use, distribution, and reproduction in any medium, provided the original work is properly cited.

Results on an optical waveguide filter operating in the near IR region are reported. The device consists of a hybrid sol-gel -based grating loaded waveguide, obtained through the merging of conventional photolithography and UV-nanoimprinting. Starting from submicrometric gratings, fabricated by electron beam lithography, a soft mould has been produced and the original structures were replicated onto sol-gel photosensitive films. A final photolithographic step allowed the production of grating-loaded channel waveguides. The devices were optically characterized by transmission measurements in the telecom range $1450-1590 \mathrm{~nm}$. The filter extinction ratio is $-11 \mathrm{~dB}$ and the bandwidth is $1.7 \mathrm{~nm}$.

\section{Introduction}

The huge demand for faster and more efficient data exchange (internet, video remote control, video private telecommunications, etc.) pushes towards photonic devices, which potentially offer much faster performances with respect to traditional interconnection and data processing based on electrons. The diffusion of optical technologies in telecommunications (TMC) requires the development of highly reliable and low-cost photonic devices (emitters, modulators, switchings, waveguides, detectors, etc.) with a high level of integration. Bragg grating optical filters are key elements in many integrated optical devices such as wavelength-division multiplexing (WDM) [1], optical fibre sensors [2-4], gainflattening filters [5], add-drop multiplexers (OADM's) [6], optical couplers, resonators, and modulators $[7,8]$.

Over the past decade, extensive research was conducted in order to identify and improve such elements, but the device fabrication is quite expensive since it requires nanometric resolution which is obtained by means of sophisticated lithographic methods (deep UV or electron beam lithography). Most of the devices developed up to now remain at laboratory level or results very expensive and not suitable for large mass production.
In order to overcome this limit, unconventional fabrication techniques have been very recently implemented with the aim of obtaining high-performance and low-cost devices [9]. In particular, nanoimprinting and soft lithography, which are nonphotolithographic processes, based on replica molding for micro- and nanofabrication [9] emerged as a valid and fast-processing alternative to traditional fabrication processes. Their cost effectiveness along with the high-quality reproduction of nanopatterned hard masters makes soft lithography an attractive technique for large-scale device production. In the very last years, several Bragg grating-based optical devices have been demonstrated by merging conventional and unconventional fabrication techniques [9-14]. Moreover, the great potential of nanoimprinting lithography has been demonstrated recently, also on large-area samples, by roll-to-roll and roll-to-plate nanoimprinting techniques [15]. Such methods are therefore suitable for exploitation on large-area and large-scale production at the same time.

In this paper, we report results on the fabrication of solgel-based optical filters operating in the IR range obtained by a combination of soft lithography and photolithographic procedures. The integration of sol-gel materials with soft lithography processes extends advantages and versatility 
of the soft lithography and allows the implementation of cheaper and more stable micro- and nanostructured optical devices.

\section{Experimental}

2.1. Materials. Polymeric and hybrid organic/inorganic materials are appropriate for optical device applications since their optical and mechanical properties can be easily tailored in order to fit a number of specific requirements. Thin films of such materials having good optical properties can be simply deposited on different substrates. Their intrinsic and related fabrication costs are competitive with respect to the widely used ones, such as $\mathrm{SiO}_{2}$, semiconductors, and (for active applications) nonlinear optical crystals. In this paper, we employed a hybrid material synthesized by means of solgel route, based on titanium propoxide $\left[\mathrm{Ti}(\mathrm{OPr})_{4}\right]$ (indicated in the following as "Ti") and 3-(trimethoxysilyl)propyl methacrylate, ("TMSPM") as precursors in weight ratio 1 to 4 , respectively. Moreover, in order to produce a photosensitive material, an amount of $4 \%$ in weight of a photoinitiator (Irgacure 184 provided by CIBA) was added. The two solgel precursors were purchased from Sigma-Aldrich and have been used without further purification. Further details related to the chemical synthesis of Ti: TMSPM (1:4) planar and channel waveguides fabrication are reported in a recent work [16].

2.2. Devices. The developed device is a hybrid sol-gel-based Bragg grating waveguide acting as an optical filter in the third telecom window. Such device has been obtained through the merging of conventional photolithography and UVnanoimprinting. The structure was fabricated onto Si (100)type substrate with thermally grown $8 \mu \mathrm{m} \mathrm{SiO} 2$ layer, acting as an optical insulating buffer.

The filtering element is composed by a ridge-type single mode waveguide with a relieve Bragg grating on its top. Once radiation at different wavelengths is coupled into the channel, the effective refractive index modulation caused by the grating allows filtering of a specific frequency exploiting the partial reflection at each periodic stack, according to the equation:

$$
\lambda_{f=2 n_{\mathrm{eff}} \Lambda}
$$

$\lambda_{f}$ is the filtered wavelength, $\Lambda$ the grating period, and $n_{\mathrm{eff}}$ the effective refractive index of the waveguide at $\lambda_{f}$. The overall reflection efficiency depends on the specific morphology of the grating, namely periodicity, depth of the grating, and length of the device.

2.3. Device Fabrication. Device fabrication is based on a replica of a hard master with desired features. Such master was realised through electron beam lithography (EBL). Our electron beam is equipped with a field emission gun with electrons accelerated at $100 \mathrm{kV}$ allowing a spatial resolution down to $20 \mathrm{~nm}$ line width; it was used in order to fabricate $\mathrm{Si}_{3} \mathrm{~N}_{4}$ hard master gratings having specific dimensions matching device design described in the following.

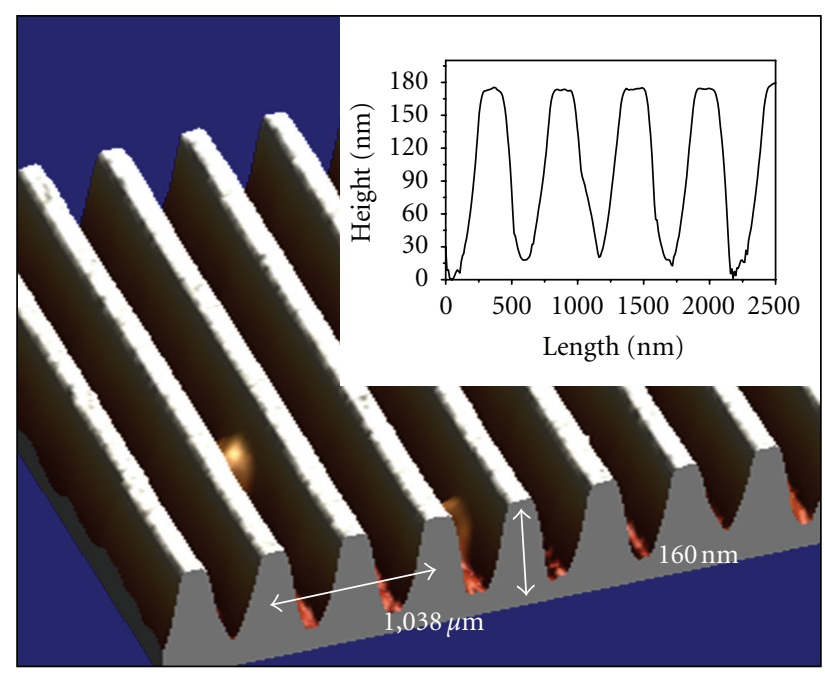

FIGURE 1: 3D AFM image of the $\mathrm{Si}_{3} \mathrm{~N}_{4}$ master grating. The insert shows the profile and the height of the periodic structures.

Morphological characterization of the surfaces nanostructured by EBL process was performed by atomic force microscopy (AFM) measurements using a VEECO Instruments AFM. An image of a planar master grating and the profile with the shape and the height of the structures are shown in Figure 1.

The first step of the procedure was the soft mould fabrication through soft lithography (SL) process using the rigid EBL master (right side of Figure 2(a)). To this end, liquid elastomer polydimethylsiloxane (PDMS Sylgard 184, Dow Corning) was directly poured onto $\mathrm{Si}_{3} \mathrm{~N}_{4}$ master. The hard master covered with PDMS solution was left in lowvacuum chamber for $30^{\prime}$ and subsequently cured at $75^{\circ} \mathrm{C}$ for further $90^{\prime}$. After this time, the soft mould was peeled-off ("de-moulding" process) the hard master (Figure 2(a)). The mould so obtained resembles with a high accuracy the hard master shape [9-12].

For grating replication, PDMS mould was placed in conformal contact with a photosensitive planar sol-gel Ti/TMSPM (1:4) film deposited by spin coating onto the $\mathrm{Si} / \mathrm{SiO}_{2}$ substrates. The film thickness was calibrated in order to obtain a single-mode waveguide at $1550 \mathrm{~nm}$.

The system is irradiated, after the contact, using an UV Xe-Hg lamp $(\lambda=280 \mathrm{~nm}-400 \mathrm{~nm})$ with intensity $I_{L}=160 \mathrm{~mW} / \mathrm{cm}^{2}$ to promote the polymerisation of the methacrylate groups of TMSPM and the densification of the material (Figure 2(b)-left side). Then the PDMS mould is gently removed leaving the periodic modulation on the hybrid sol-gel film surface (right side of Figure 2(b)).

In order to define the correct UV exposure time for matrix densification, previous tests were performed on planar films with similar thickness. The refractive index $n$ was measured by means of a variable angle spectroscopic ellipsometer (VASE J.A. Woollam Co.). Changes of the optical properties of the material as a function of UV irradiation have been recently studied in detail [10, 13]. After 10 seconds of UV irradiation at $160 \mathrm{~mW} / \mathrm{cm}^{2}$, following 

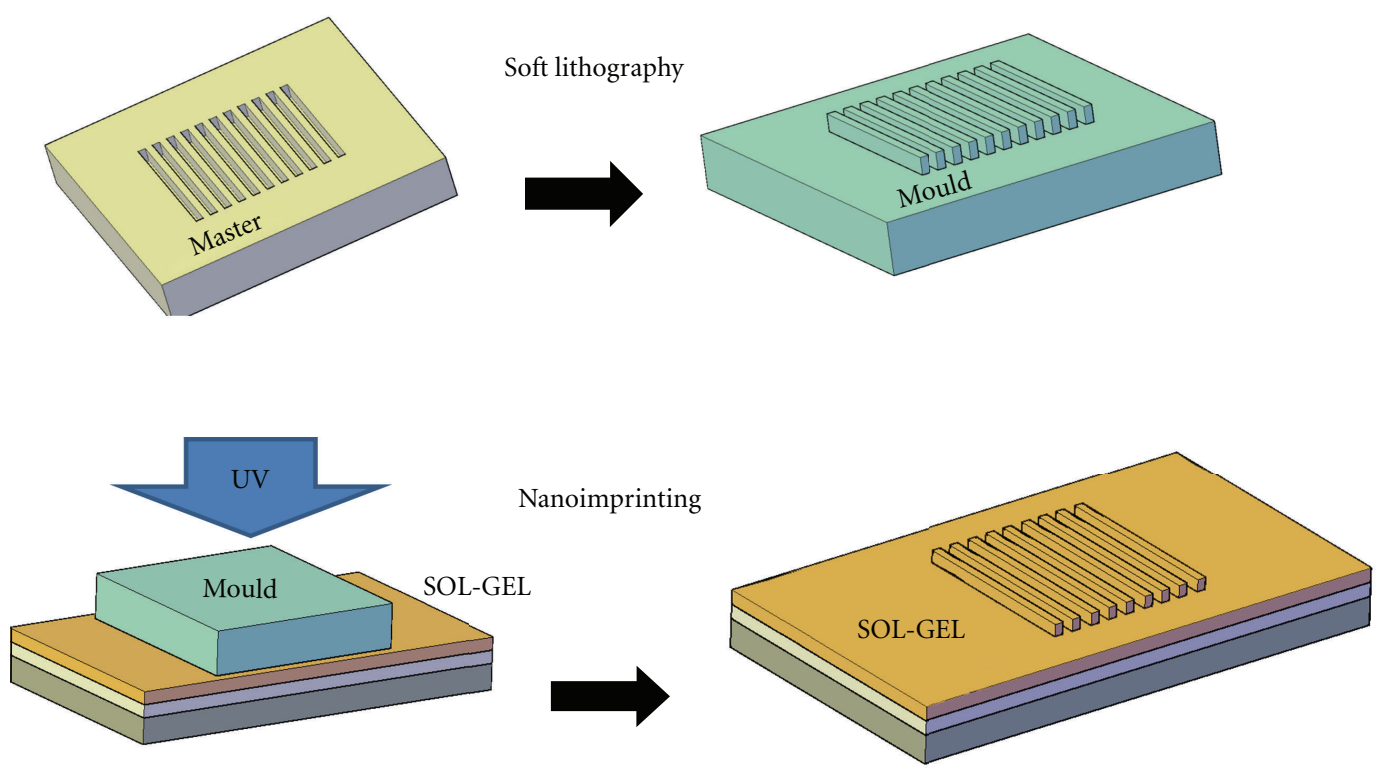

Conventional lithography

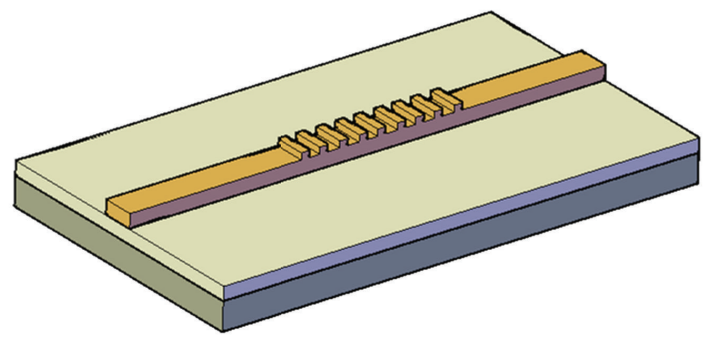

Figure 2: Main device fabrication steps.

results of previous FTIR studies [16], the polymerization degree of TMSPM sol-gel precursor reaches a plateau value and, similarly, refractive index increases from 1.49 up to a value of 1.53. Any further irradiation does not affect index of refraction, indicating a complete polymerization of the organic compound.

The final step of the fabrication process (Figure 2(c)) was the channel waveguide definition: a photoresist (PR) (S1813 Shipley) has been poured on top of the patterned sol-gel sample and spun at $3000 \mathrm{rpm}$ in order to obtain a $1.3 \mu \mathrm{m}$ PR film. Then the samples underwent a prebake step at $90^{\circ} \mathrm{C}$ on a hot plate for 5 minutes in order to evaporate residual solvents. UV exposure through a prefabricated chromium/quartz mask was performed by means of a mask aligner in order to define channel waveguides perpendicularly oriented with respect to the Bragg gratings. A development step was then accomplished in Shipley developer MF319 to remove the unexposed parts. Subsequently, a hard bake step followed and samples were left at $125^{\circ} \mathrm{C}$ for $20^{\prime}$.

The lateral definition of the waveguide was finally accomplished by a reactive ion etching (RIE) process. A gas mixture SF6-Ar with a working pressure of 60 mTorr has been used and the RIE power density was $0.33 \mathrm{~W} / \mathrm{cm}^{2}$. The measured etching rate was $\sim 60 \mathrm{~nm} / \mathrm{min}$. The dimensions of the final device were $6 \mu \mathrm{m}$ width and a length of $3 \mathrm{~cm}$. The smoothness of the side walls and the good definition of the channels can be appreciated in Figure 3 where the 3 -dimensional AFM image of the final Ti/TMSPM $(1: 4)$ wavelength filter device is reported. Channel thickness has been measured by means of an $\alpha$-step profiler (Techno Instruments). The height of the structure was about $1.8 \mu \mathrm{m}$.

\section{Results and Discussion}

Our goal was the manufacturing of an integrated filter operating in the third window of telecommunication band. In order to fulfil this intention, the dimension of the channel waveguide (width and height), the grating depth, and the grating period have to be accurately chosen, taking into account the refractive index $n$ of the Ti/TMSPM. On this regard, preliminary computer simulations have been carried out. 


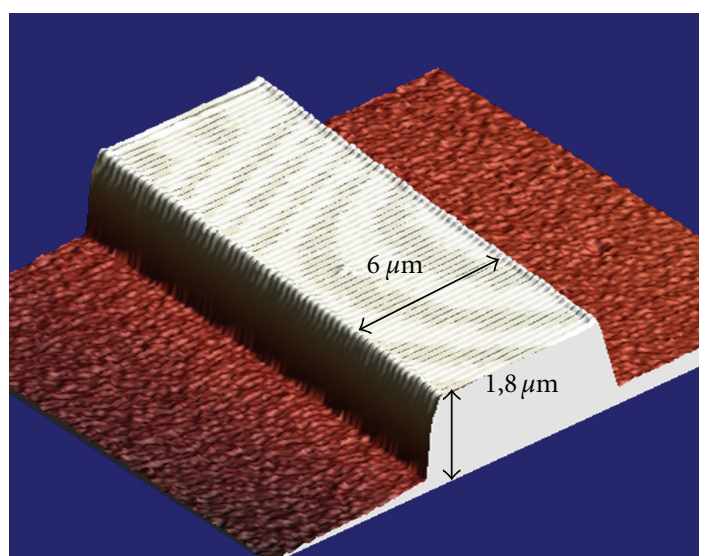

FIgUre 3: AFM image of a Ti/TMSPM (1:4) wavelength filter. Channel waveguide thickness and width were 1.8 and $6 \mu \mathrm{m}$, respectively.

First of all, the value of $n_{\text {eff }}$ in (1) has to be correctly calculated. Such value depends on the material but also on the width and height of the channel waveguide. Its value was estimated by finite element method (FEM) numerical model considering that our structure has a fixed width of $6 \mu \mathrm{m}$ and a height that varies periodically with the periodicity of the imprinted grating. For such reason, two different thickness of channel have been considered, namely, 1800 and $1960 \mathrm{~nm}$. These values are related to the layer thickness of the initial planar waveguide and to the maximum grating depth attainable with the hard master defined with EBL $(160 \mathrm{~nm})$. We calculated a minimum value of effective refractive index (corresponding to a waveguide without grating) and a maximum value corresponding to a thicker waveguide including $160 \mathrm{~nm}$ extralayer (corresponding to the height of the grating). The proper $n_{\text {eff }}$ value used in (1) ( $n_{\mathrm{eff}}=1.489$ at $1550 \mathrm{~nm}$, TE polarization) was the average value of the two above mentioned calculations. Inserting this value in the equation, it results that a grating period of $519 \mathrm{~nm}$ should generate a channel waveguide filter acting at $1544 \mathrm{~nm}$. For these reasons, the EBL master was designed and fabricated with a periodicity of $520 \mathrm{~nm}$ and a depth of $160 \mathrm{~nm}$.

The morphology of the final device obtained applying the UV-nanoimprinting and the subsequent ridge channel RIE definition is shown in Figure 3. Comparison of the morphology of the hard master and final nanostructured solgel layer shows that no appreciable shrinkage occurs.

To test our device, a collimated TE-polarized beam coming from a pigtailed, tuneable diode laser source (Nettest, Tunics-Plus) has been coupled by end fire into our device. In order to obtain low-coupling losses, cleaving procedure was used for producing a clean in- and endface of the waveguide device. Light collected at the end of the channel waveguide by a 20x microscope objective was focused onto a germanium photodiode.

Transmission test of the channel waveguide has been performed as a function of wavelength by scanning the laser light in a range between $1450 \mathrm{~nm}$ to $1590 \mathrm{~nm}$ in order to

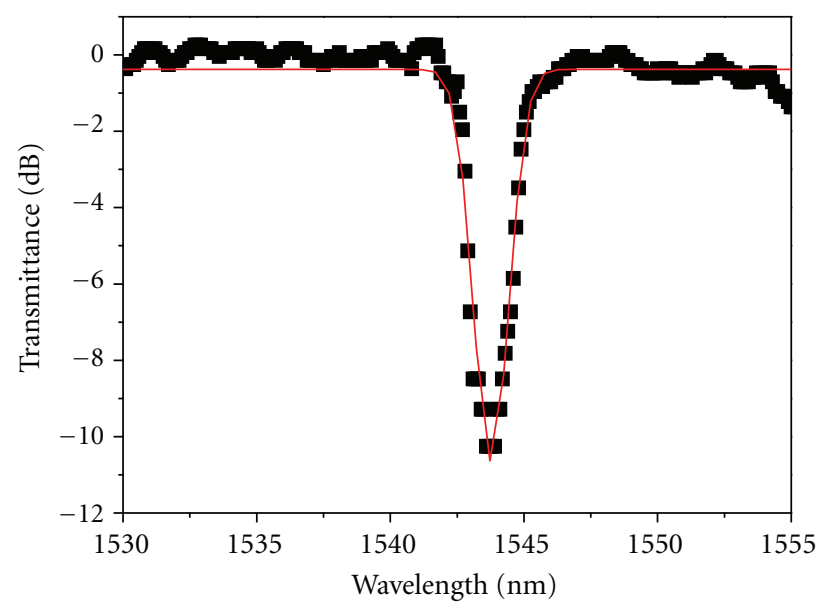

FIgURE 4: Transmission intensity as a function of the wavelength showing filtering effect of a Bragg grating-loaded Ti/TMSPM $(1: 4)$ sol-gel waveguide.

evaluate the transmittance of the device. In Figure 4, the transmittance spectrum of the system is reported. Filtering effect has been obtained at $1543.7 \mathrm{~nm}$. In the figure is also reported (solid line) a Lorentzian fit of the experimental data showing that the transmission dip is about $-11 \mathrm{~dB}$ and the bandwidth at full width half maximum (FWHM) is $1.7 \mathrm{~nm}$.

Experimental data are fully consistent with the theoretical design and computer simulations. The high-quality performances of our filter show that the UV-nanoimprinting represents a valid route to produce devices with characteristics as good as that obtained by direct EBL fabrication process. Moreover, this technique is suitable for large-scale production due to the intrinsic reproducibility of the process where the same hard master can be used many times.

\section{Conclusion}

Ti-based wavelength filter, fabricated by the merging of conventional photolithography and UV-nanoimprinting, has been produced. The process was applied for the fabrication of a channel waveguide based on hybrid sol-gel material with Bragg grating imprinted on its top, thus obtaining an efficient IR-integrated optical filter with rejection up to $11 \mathrm{~dB}$ and bandwidth of $1.7 \mathrm{~nm}$.

\section{Acknowledgments}

The authors are deeply indebted to Dr. Glauco Stracci for his experienced technical assistance. This paper was supported in part by the Italian Ministry of University through the FIRB RBNE033KMA Italian project "Molecular and hybrid organic/inorganic nanostructures for photonics" and by the CARIPLO foundation through the project number 20100525 . 


\section{References}

[1] J. Hübner, D. Zauner, and M. Kristensen, "Strong sampled bragg gratings for WDM applications," IEEE Photonics Technology Letters, vol. 10, no. 4, pp. 552-554, 1998.

[2] G. B. Tait, "Vernier-effect optical interrogation technique for fiber Bragg grating sensors," Applied Optics, vol. 46, no. 28, pp. 6879-6884, 2007.

[3] W. Liang, Y. Huang, Y. Xu, R. K. Lee, and A. Yariv, "Highly sensitive fiber Bragg grating refractive index sensors," Applied Physics Letters, vol. 86, no. 15, Article ID 151122, 3 pages, 2005.

[4] T. Pustelny, I. Zielonka, C. Tyszkiewicz, P. Karasiński, and B. Pustelny, "Impressing technology of optical Bragg's gratings on planar optical sol-gel waveguides," Opto-Electronics Review, vol. 14, no. 2, pp. 161-166, 2006.

[5] A. E. Lobo, J. A. Besley, and C. Martijn de Sterke, "Gainflattening filter design using rotationally symmetric crossed gratings," Journal of Lightwave Technology, vol. 21, no. 9, pp. 2084-2088, 2003.

[6] L. Eldada, S. Yin, C. Poga, C. Glass, R. Blomquist, and R. A. Norwood, "Integrated multichannel OADM's using polymer bragg grating MZI's," IEEE Photonics Technology Letters, vol. 10, no. 10, pp. 1416-1418, 1998.

[7] Y. Huang, G. T. Paloczi, A. Yariv, C. Zhang, and L. R. Dalton, "Fabrication and replication of polymer integrated optical devices using electron-beam lithography and soft lithography," Journal of Physical Chemistry B, vol. 108, no. 25, pp. 8606-8613, 2004.

[8] D.-H. Kim, W.-J. Chin, S.-S. Lee, S.-W. Ahn, and K.-D. Lee, "Tunable polymeric Bragg grating filter using nanoimprint technique," Applied Physics Letters, vol. 88, no. 7, Article ID 071120, 3 pages, 2006.

[9] C. M. Sotomayor Torres, Alternative Lithography: Unleashing the Potentials of Nanotechnology, Kluwer Academic/Plenum, New York, NY, USA, 2003.

[10] P. Prosposito, M. Casalboni, E. Orsini, C. Palazzesi, and F. Stella, "UV-nanoimprinting lithography of Bragg Gratings on hybrid sol-gel based channel waveguides," Solid State Sciences, vol. 12, no. 11, pp. 1886-1889, 2010.

[11] Z. Li, Y. Gu, L. Wangs et al., "Hybrid nanoimprint-soft lithography with sub-15 nm resolution," Nano Letters, vol. 9, no. 6, pp. 2306-2310, 2009.

[12] A. Kocabas and A. Aydinli, "Polymeric waveguide Bragg grating filter using soft lithography," Optics Express, vol. 14, no. 22, pp. 10228-10232, 2006.

[13] A. Moujoud, H. J. Kim, S. H. Kang et al., "Double component long period waveguide grating filter in sol-gel material," Optics Express, vol. 15, no. 23, pp. 15147-15153, 2007.

[14] W. C. Chuang, C. K. Chao, and C. T. Ho, "Fabrication of highresolution periodical structures on polymer waveguides using a replication process," Optics Express, vol. 15, no. 14, pp. 86498659, 2007.

[15] S. H. Ahn and L. J. Guo, "Large-area roll-to-roll and roll-to-plate Nanoimprint Lithography: a step toward highthroughput application of continuous nanoimprinting," ACS Nano, vol. 3, no. 8, pp. 2304-2310, 2009.

[16] X. Le Guével, C. Palazzesi, P. Prosposito, G. Della Giustina, and G. Brusatin, "Influence of chelating agents on the photopolymerization of hybrid Ti-based waveguides," Journal of Materials Chemistry, vol. 18, no. 30, pp. 3556-3562, 2008. 

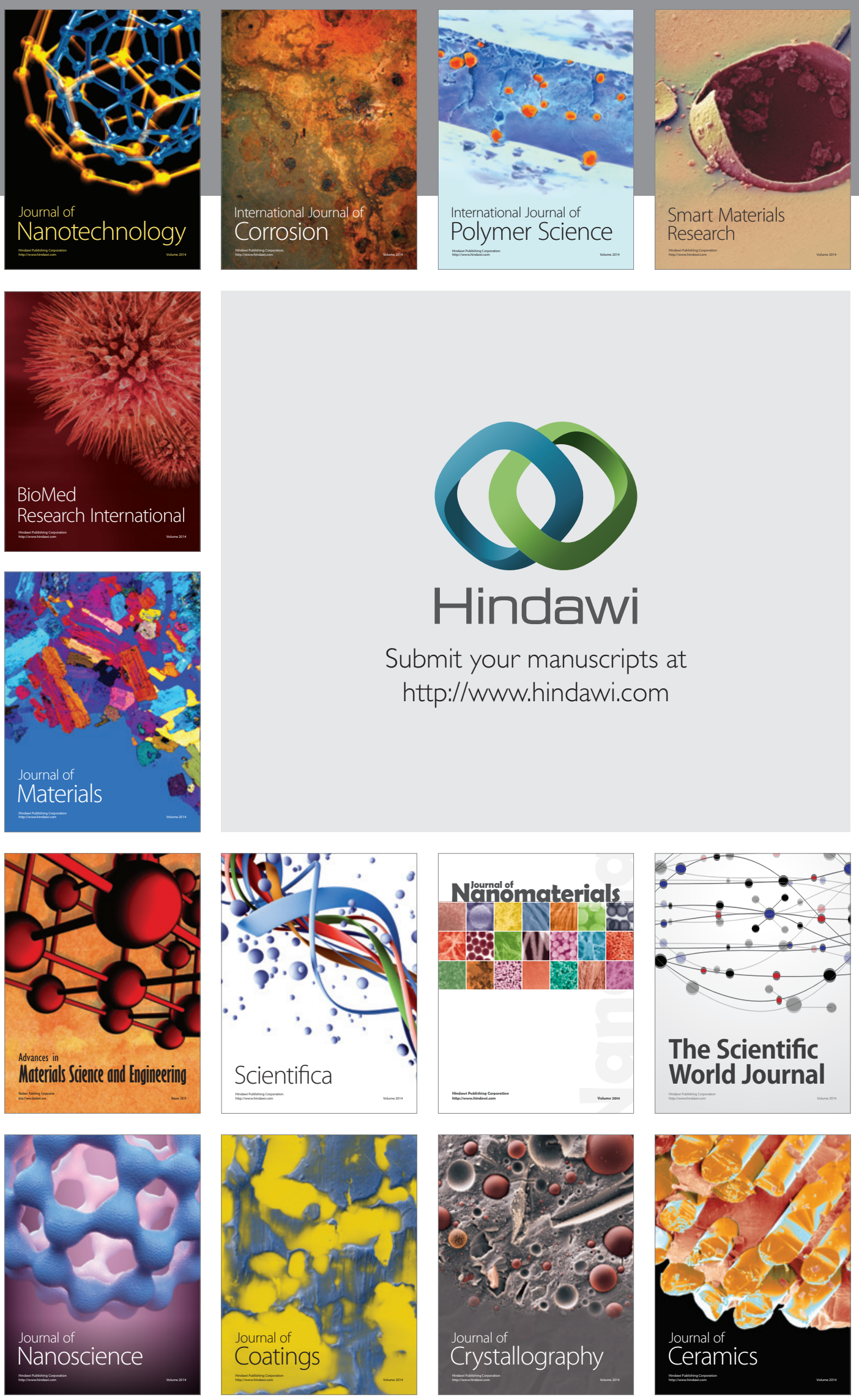

The Scientific World Journal

Submit your manuscripts at

http://www.hindawi.com

\section{World Journal}

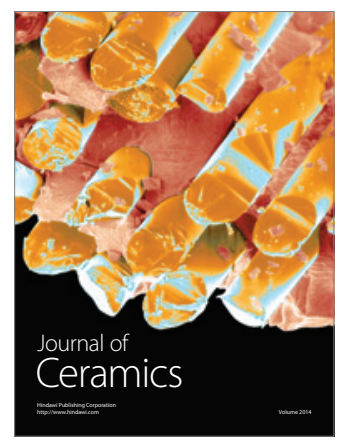

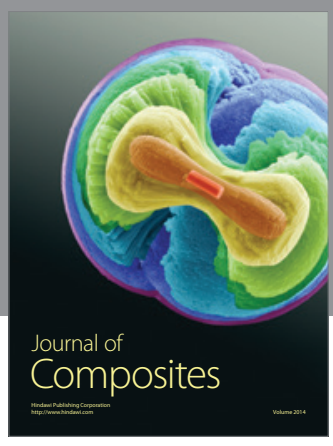
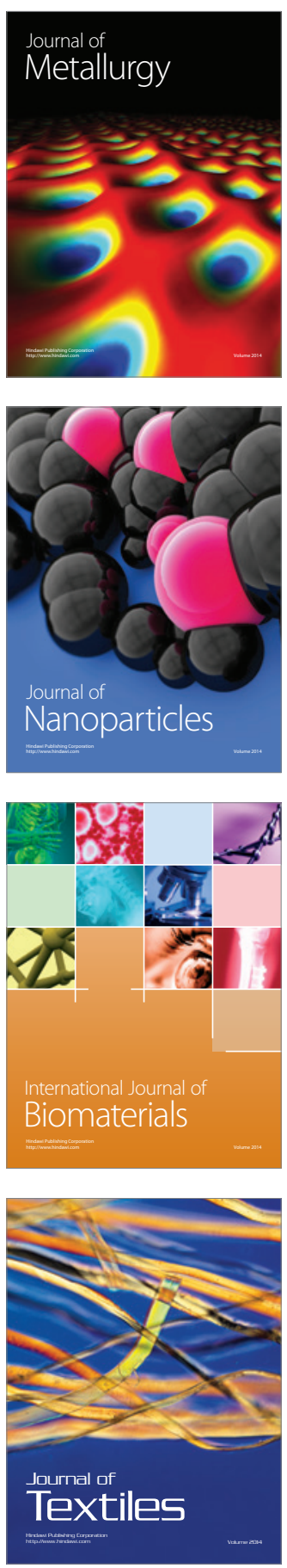